\title{
The Correlation between Left Atrial Function by Speckle Tracking and Stroke Risk in Patients with Persistent Atrial Fibrillation
}

H.M.AboEl Enin, A.Y.Ahmed, A.M.Sabry and M.A.Ammar

Cardiovascular Dept., Faculty of Medicine, Benha Univ., Benha, Egypt

E-Mail: Noormero.ama@gmail.com

\begin{abstract}
The primary persistent Atrial fibrillation (AF) is the most common sustained arrhythmia in the general population; its prevalence increases with age and is generally associated with increased mortality. To decide the relationship between's left atrial capacity by dot following echocardiography and stroke chance in understanding with industrious atrial fibrillation. This examination included 100 patients with industrious AF convoluted with stir alluded to the Cardiology Department at Benha University Hospital for echocardiographic appraisal, who were contrasted and 100 patients with determined AF not entangled with stroke of coordinated age and sexual orientation. The collector administrator trademark (ROC) bend was utilized to test demonstrative estimation of different echocardiographic boundaries models in the connection between's left atrial capacity by dot following and stroke hazard in patients with tireless atrial fibrillation and indicated that the estimation of LA strain $<22.5$ was appeared to have the best symptomatic precision with (affectability $=$ $82 \%$; explicitness $=60 \%$ ) in foreseeing the stroke chance in atrial fibrillation understanding with AUC.871. It is reasoned that spot following investigations of the left chamber may offer important data on which patients would profit the most from delayed cardiovascular observing for distinguishing proof of PAF.
\end{abstract}

Keywords: Atrial Function, Speckle Tracking, Atrial Fibrillation.

\section{Introduction}

Atrial fibrillation brings about auxiliary, hemodynamic and electrical outcomes [1].

Patients with industrious $\mathrm{AF}$ have a considerable danger of stroke, which is changed by the nearness or the nonattendance of a few hazard factors [2].

As counteraction of stroke/foundational embolism is by a wide margin the most significant part of clinical administration of atrial fibrillation (AF), an approved CHA2DS2VASc score framework is currently suggested as a Class 1A sign for chance appraisal of stroke [3].

There is no denying the way that all the components of the CHA2DS2VASc score alone or in mix can shape a blood clot to cause calamitous occasions as all these clinical variables are conceivably thrombogenic [4].

Nearness of impeded LA twisting boundaries assessed by 2D spot following technique were related with the nearness of LAA blood clot. LAA being a piece of LA, its capacities are believed to be reflected by LA basic and useful boundaries. LA capacities can be isolated into 3 stages in a heart cycle. These are (1) a repository stage accepting blood from the pneumonic veins during ventricular systole; V. Fuster et al. [2] a detached channel stage during early diastole, and $\mathrm{P}$. Kirchhof et al.[3] a contractile stage during late diastole. Customarily, LA distance across and volume and transmitral and aspiratory vein Doppler speeds were utilized for assessment of these capacities. As of late, new techniques that can quantify myocardial misshapening, for example, CTDI strain and 2D STE strain have been applied. CTDI has various restrictions, for example, point reliance, signal ancient rarities, and constrained reproducibility; then again, STE is edge autonomous and could be utilized to gauge territorial as well as worldwide atrial strain [4].

Propelled age, steady AF, expanded LA volume, mitral stenosis, LV brokenness, and prosthetic valves were related with traded off LAA capacities and the nearness of unconstrained reverberation differentiate (SEC) or blood clot [2].

During the previous quite a long while, strain and strain rate imaging have developed as a quantitative strategy to appraise myocardial capacity and contractility precisely. Two-measurement strain imaging is another echocardiographic procedure for getting strain and strain rate estimations. It examines movement by tissue Doppler in the ultrasonic picture in two measurements [5].

The investigation of left a preliminary (LA) strain is another instrument that can be utilized to assess LA work [3].

\section{Aim of The Work}

The correlation between left atrial function by speckle tracking echocardiography and stroke risk in patient with persistent atrial fibrillation.

\section{Patients and methods}

Study design

It was a single center, cross-sectional, comparative study that was conducted at cardiology department at "Benha University Hospital".

\section{Patient selection}

This study included100 patients with persistent AF complicated with stoke referred to the Cardiology Department at Benha University Hospital for echocardiographic assessment, who were compared with 100 patients with persistent AF not complicated with stroke of matched age and gender.

\section{Inclusion criteria}

Patients with persistent atrial fibrillation type in which episode of irregular atrial rhythm lasts for longer than 12 months, cardioversion can be used to stop the episode, as it is unlikely to stop on its own. 
Exclusion criteria

Patients who had one or more of the following criteria were be excluded from the study:

1- Patients with primary valvular disease.

2- Patients with congenital heart disease.

3- Patients with primary myocardial disease.

4- Patients with pericardial disease.

5- Uncontrolled AF.

6- Patient refusal.

7- Poor echogenicity.

8- Mitral annular calcification.

\subsection{Methods}

The included patients were subjected to the following

1. History

- Age and gender.

- The presence or absence of comorbid conditions as diabetes, hypertension, smoking, heart failure, a history of stroke and vascular diseases, including coronary artery disease, heart attack (myocardial infarction) and peripheral arterial disease were taken into account to risk stratify the participants according to CHA2DS2-VASccriteria.

2. Full clinical examination

Including heart rate, rhythm, systolic blood pressure (SBP), diastolic blood pressure (DSP), chest and heart auscultation.

\section{Investigations}

\section{Electrocardiography}

Twelve lead surface ECG was done with a Cardio Fax C machine, voltage $10 \mathrm{~mm} / \mathrm{mv}$, velocity $25 \mathrm{~mm} / \mathrm{sfor}$ each patient to confirm the presence of atrial fibrillation.

\section{Echocardiography}

Two-dimensional echocardiography and Doppler examination will be performed with EPIQ7CPHILLIPS Ultrasound Machine with a multi frequency 1.74MHzTransducer of the 's5-1' type and conducted to a single-lead ECG. All examinations were performed with the patients in the standard left lateral position.

\section{Two-dimensional echocardiography}

\section{- LA dimensions}

The LA size was measured at the end-ventricular systole when the LA chamber is at its greatest dimension, in parasternal long-axis view (anteriorposterior diameter) and in apical 4-chamber view (longitudinal and transverse diameters) [6].

\section{- LA volumes}

LA latent volumes comprise of insignificant LA volume ( $\mathrm{V}$ min), estimated not long before the conclusion of the mitral valve in end-diastole; and maximal LA volume ( $\mathrm{V} \max$ ), estimated not long before the opening of the mitral valve in end-systole [6].

The distinction among most extreme and least LA volume separated by the greatest LA volume is utilized to identify uninvolved atrial discharging division.
- LV volumes and Left ventricular ejection fraction

Global LV function was assessed by measuring LV end-diastolic volume (LVEDV), LV end-systolic volume (LVESV) and LVEF from the conventional apical 2- \& 4-chamber images, using the biplane Simpson's method [7].

\section{Pulsed doppler transmitral flow}

Mitral inflow designs by beat wave Doppler assessment show uninvolved ventricular filling in early diastole (E wave). The test volume is put at the tips of the mitral pamphlets in the apical four-chamber see.

\section{Tissue doppler imaging}

Beat wave tissue Doppler imaging (TDI) was acted in the apical perspectives to secure mitral annular speeds. Estimations incorporated the systolic (S) and early diastolic $\left(\mathrm{E}^{\prime}\right)$ [8].

The beat wave TDI test volume was put on the mitral annulus in the apical four-and two-chamber sees.

\section{Strain doppler method}

For strain Doppler assessment, ongoing 2D shading Doppler myocardial imaging information are recorded from the LA, utilizing standard apical perspectives at a high casing rate $(>180 \mathrm{fps})$. The information were put away in computerized position and examined disconnected by devoted programming that permits ascertaining neighborhood top systolic strain (9). Four fragments were assessed in apical 4 and apical 2 perspectives.

\section{Speckle tracking analysis}

Two-dimensional STE examination will be performed disconnected on standard 2D grayscale pictures from apical four-chamber, two-chamber, and three-chamber sees for LV GLS and from four-chamber, two-chamber sees for worldwide or mean PALS. In the investigation of the left chamber, the $3 \mathrm{CH}$ view isn't considered on account of the nearness of the aortic valve and climbing aorta.22 Data were gained with a casing pace of in any event 60 edges for every second during breath hold. The outskirt of the LV and LA endocardium was be physically followed at end systole in two separate examinations. In the two cases, the district of intrigue will be acclimated to the divider thickness (enlarged or limited). Strain estimations of the LV were be estimated consequently in 18 sections, and the product created the normal LVGLS. Additionally, we estimated PALS (LA extending) in two habits by the utilization of the worldwide strain bend (GPALS).

\section{Measurable examination}

The gathered information were summed up regarding mean \pm standard deviation (SD) and range for quantitative information and recurrence and rate for subjective information.

Examinations between the investigation bunches were done utilizing the Chi-square test $\left(\chi^{2}\right)$ and Fisher's 
Exact Test (FET) to think about extents as proper. The Student t-test $(\mathrm{t})$ was utilized to identify mean contrast between AF patients and controls with respect to the ordinarily conveyed information. While the Mann Whitney test (z) was utilized to think about two nontypically circulated information. One route Analysis Of Variance (ANOVA; F) was utilized to look at multiple gatherings for typically disseminated information.

Pearson connection coefficient (r) and Spear man relationship coefficient (rho; $\rho$ ) were utilized to test for the relationship between's CHA2DS2-VAScscores and regularly and non-typically circulated Echo boundaries individually.

The recipient administrator trademark (ROC) bend was utilized to test analytic execution of systolic LA strain in foreseeing CHA2DS2-VASc score equivalent to or more than 2 among AF patients. The best cutoff point and the comparing affectability and explicitness and region under the bend (AUC) were assessed.

After the figuring of every one of the test insights, the comparing dissemination tables were counseled to get the "P" (likelihood esteem). Factual criticalness was acknowledged at $\mathrm{P}$ esteem $<0.05(\mathrm{~S})$. A P esteem $<0.001$ was considered profoundly noteworthy (HS) while a $\mathrm{P}$ esteem >0.05 was considered non-critical (Stata 2009).

$$
\begin{gathered}
x^{2}=\sum \frac{(\text { observed }-\exp \text { ected })^{2}}{\text { Expected }} \\
\text { Expected }=\frac{\text { col.total } \times \text { row total }}{\text { Grand total }}
\end{gathered}
$$

A P value $<0.05$ was considered statistically significant $(*)$ while $>0.05$ statistically insignificant $\mathrm{P}$ value $<0.01$ was considered highly significant $(* *)$ in all analyses.

\section{Results}

It is a single centre, cross sectional, comparative study, that was conducted at cardiology department at "Benha University hospital".

This study included 100 patients with persistent AF complicated with stoke referred to the Cardiology Department, Benha University Hospital, who were compared with 100 patients with persistent AF not complicated with stroke with their age and gender matching with group 1 .
The clinical data were recorded on a report form. These data were tabulated and analyzed using the computer program SPSS (Statistical package for social science) version 20 to obtain:

\section{1- Descriptive data}

Descriptive statistics were calculated for the data in the form of:

1 - Mean and standard deviation $( \pm S D)$. Median and interquartile range (IQR) for quantitative data.

2- Frequency and distribution for qualitative data.

\section{3- Analytical statistics}

In the statistical comparison between the different groups, the significance of difference was tested using one of the following tests after establishing their non normality by K-S test (One-Sample KolmogorovSmirnov Test) of normality.

1 - Student's $t$-test and Mann-Whitney test:- Used to compare mean of two groups of quantitative data of parametric and non-parametric respectively.

2 - Inter-group comparison of categorical data was performed by using chi square test ( $X^{2}$-value).

\section{Data management}

\section{Patient demographics and personal data}

There was no significant statistical difference between the 2 groups as regarding to the age ( $\mathrm{p}$ value $>$ 0.16 ) and gender ( $\mathrm{p}$ value $>0.32$ ).

In patients of group I, 60 patients $(60 \%)$ were males and 40 patients $(40 \%)$ were females and their age ranged from 50-68 years, with a mean age of 58years.

In patients of group II, 55 patients $(55 \%)$ were males and 45patients $(45 \%)$ were females and their age ranged from 49-65 years, with a mean age of 57.5 years Table (1).

Table (1) Comparison between both groups regarding personal data (age \& gender).

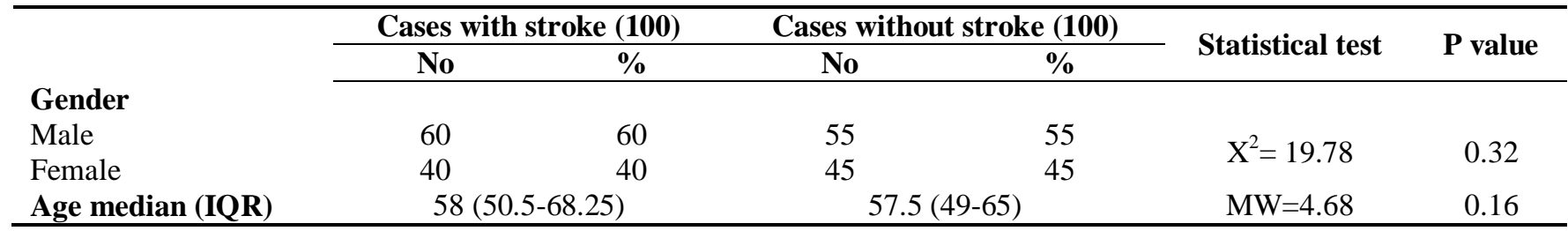

\section{Past history and risk factors}

Patients with Past history of hypertension and diabetes were more prevalent in patients with group I compared with group II patients (55 vs $25, \mathrm{p}=.001$ and 45 vs $25, \mathrm{p}=.003$, respectively).

There was no significant statistical difference between the two groups regarding number of patients with past history of heart failure, peripheral vascular disease (50 vs 55, $\mathrm{p}=.48$ and 25 vs $30, \mathrm{p}=.53$, respectively) Table (2). 
Table (2) Comparison between cases with and without stroke regarding risk factors.

\begin{tabular}{|c|c|c|c|c|c|c|}
\hline & \multicolumn{2}{|c|}{ Cases with stroke (100) } & \multicolumn{2}{|c|}{ Cases without stroke (100) } & \multirow{2}{*}{ Statistical test } & \multirow{2}{*}{$P$ value } \\
\hline & No & $\%$ & No & $\%$ & & \\
\hline \multicolumn{7}{|l|}{$\mathbf{H F}$} \\
\hline Present & 50 & 50 & 55 & 55 & \multirow{3}{*}{$X^{2}=0.50$} & \multirow{3}{*}{0.48} \\
\hline Absent & 50 & 50 & 45 & 45 & & \\
\hline HTN & & & & & & \\
\hline Present & 55 & 55 & 25 & 25 & \multirow{3}{*}{$X^{2}=18.75$} & \multirow{3}{*}{$<0.001 * *$} \\
\hline Absent & 45 & 45 & 75 & 75 & & \\
\hline DM & & & & & & \\
\hline Present & 45 & 45 & 25 & 25 & \multirow{3}{*}{$X^{2}=8.79$} & \multirow{3}{*}{$0.003 * *$} \\
\hline Absent & 55 & 55 & 75 & 75 & & \\
\hline Vascular & & & & & & \\
\hline Present & 25 & 25 & 30 & 30 & \multirow{2}{*}{$X^{2}=0.63$} & \multirow{2}{*}{0.53} \\
\hline Absent & 75 & 75 & 70 & 70 & & \\
\hline
\end{tabular}

\section{Clinical characteristics}

Heart rate and systolic blood pressure (SBP) were significantly increased in patients of group 1 (84 vs 77.5 bpm, $\mathrm{p}=.003$ and 125 vs $120 \mathrm{mmHg}, \mathrm{p}=.018$, respectively).
There was no significant statistical difference between the two groups regarding diastolic blood pressure (DBP) (70 vs 70 mmHg, p =.194) Table( 3).

Table (3) Comparison between cases with and without stroke according systolic, diastolic blood pressure and heart rate at presentation.

\begin{tabular}{ccc}
\hline & \multicolumn{2}{c}{ Cases with stroke (100) } \\
& Median & IQR \\
\hline SBP & 125 & $110-138.75$ \\
DBP & 70 & $62.5-80$ \\
Pulse & 84 & $71.25-90$ \\
\hline \multicolumn{4}{l}{ Echocardiographic parameter } \\
Conventional Echocardiographic parameters
\end{tabular}

On comparing conventional echocardiographic measurements in the two studied groups, Transverse LA diameter and LA minimal volume were significantly increased in patients of group I compared with group II patients $(4.6 \mathrm{~cm}(4.4-4.88)$ vs.4.5cm (4-4.7), $\mathrm{p}<0.001$ and $41 \mathrm{~cm}(38.25-46)$ vs $39.5 \mathrm{~cm}(33-44.75), \mathrm{p}=.028$, respectively).

LA emptying fraction was significantly reduced in patients of group I compared with group II patients (29.5 (26-34.75) vs. 35.5 (30.25-45), $\mathrm{P}<0.001)$.

Systolic left atrial strain was significantly reduced in patients of group I compared with patients of group II (8.9 (7.78-9.85) vs 14.5 (12-18.5), p <0.001).

There was no significant difference as regarding the anteroposterior (AP) LA diameter and longitudinal LA diameter between the comparative groups $(3.95 \mathrm{~cm}(3.63-$ $4.28)$ vs. $3.9 \mathrm{~cm}(3.63-4.35), \mathrm{P}=0.93$ and $5.1(4.73-5.8)$ vs 5.1 (4.63-5.8), $\mathrm{P}=0.98$, respectively).

Also, the LA volume maximal was of no significant difference in patients of group I compared with group II patients. (74.5 $\mathrm{ml}$ (69.25-78.75) vs. $74.5 \mathrm{ml}$ (66.25-78).

Left ventricular end diastolic volume (LVEDV) and left ventricular end systolic volume (LVESV) were of no significant difference in both groups (131.5 (121.25-

$\begin{array}{lcc}\text { roke (100) } & \text { Statistical test (MW) } & \text { P value } \\ \text { IQR } & 2.37 & 0.018^{*} \\ 00-133.75 & 1.3 & 0.194 \\ 60-78.75 & 2.95 & 0.003^{* *} \\ 70-90 & \end{array}$

$156.75)$ vs. $143 \mathrm{ml}(99-152.25), \mathrm{p}=0.58$ and 52 (38.7555.75), vs 50 (44-64), $\mathrm{p}=.34$, respectively).

Left ventricular ejection fraction (LVEF) was of no significant difference in patients of group I compared with patients of group II $(50.8 \pm 9.39$ vs. $51.2 \pm 8.99$, p $=.76$ ).

By TDI The mitral E/e" ratio was of no significant difference in patients with group I compared with group II patients $(10.73 \pm 2.57 \mathrm{vs} .11 .16 \pm 1.66$, $\mathrm{p}$ value 0.198$)$.

Regarding to peak E was of no significant difference in patients with group I compared with group II patients (75 (65-83.25) vs76.5 (65-83.75), early diastolic mitral annular velocity (E") (7.75 (6.93-8.48) vs. 8 (7-8.6), $\mathrm{p}=$ 0.67 ) Table (4).

There was significant decrease in LA strain with in patients with CHA2DS2-VASc score > 2 compared to patients with CHA2DS2-VASc score < $2(16(14-20)$ vs 30 (21-34), p <.001) Table (5).

The receiver-operator characteristic (ROC) curve was used to test diagnostic value of various echocardiographic parameters models in the correlation between left atrial function by speckle tracking and stroke risk in patients with persistent atrial fibrillation The value of LA strain $<22.5$ was shown to have the best diagnostic accuracy with (sensitivity $=82 \%$; specificity $=60 \%$ ) in predicting the stroke risk in atrial fibrillation patient with AUC.871 Table (6). 
Table (4) Comparison between cases with and without stroke according to echocardiographic parameters.

\begin{tabular}{|c|c|c|c|c|c|c|}
\hline & \multicolumn{2}{|c|}{ Cases with stroke (100) } & \multicolumn{2}{|c|}{$\begin{array}{c}\text { Cases without stroke } \\
(\mathbf{1 0 0})\end{array}$} & \multirow{2}{*}{$\begin{array}{l}\text { Statistical test } \\
\text { (MW) }\end{array}$} & \multirow{2}{*}{$P$ value } \\
\hline & Median & IQR & Median & IQR & & \\
\hline Antero-post LA diameter & 3.95 & $3.63-4.28$ & 3.9 & $3.63-4.35$ & 0.09 & 0.93 \\
\hline $\begin{array}{l}\text { Longitudinal LA } \\
\text { diameter }\end{array}$ & 5.1 & $4.73-5.8$ & 5.1 & $4.63-5.8$ & 0.03 & 0.98 \\
\hline Transverse LA diameter & 4.1 & $3.73-4.58$ & 4.65 & $4.43-4.98$ & 5.36 & $<0.001 * *$ \\
\hline MAX volume & 74.5 & $69.25-78.75$ & 74.5 & $66.25-78$ & 0.67 & 0.50 \\
\hline MIN volume & 41 & $38.25-46$ & 39.5 & $33-44.75$ & 2.2 & $0.028 *$ \\
\hline LAEF & 29.5 & $26-34.75$ & 35.5 & $30.25-45$ & 5.96 & $<0.001 * *$ \\
\hline Peak E & 75 & $65-83.25$ & 76.5 & $65-83.75$ & 0.43 & 0.67 \\
\hline $\mathbf{E}$ & 7.75 & $6.93-8.48$ & 8 & $7-8.6$ & 1.81 & 0.07 \\
\hline E/e" Mean \pm SD & \multicolumn{2}{|c|}{$10.73 \pm 2.57$} & \multicolumn{2}{|c|}{$11.16 \pm 1.66$} & St $\mathrm{t}=1.39$ & 0.198 \\
\hline SLA strain & 8.9 & $7.78-9.85$ & 14.5 & $12-18.5$ & 9.08 & $<0.001 * *$ \\
\hline LA strain (\%) & 18 & $14-21$ & 21 & $17-31.75$ & 4.04 & $<0.001 * *$ \\
\hline LVESV & 52 & $38.75-55.75$ & 50.5 & $44-64$ & 0.95 & 0.34 \\
\hline LVEDV & 131.5 & $\begin{array}{l}121.25- \\
156.75\end{array}$ & 143 & $99-152.25$ & 0.55 & 0.58 \\
\hline EF\% Mean \pm SD & \multicolumn{2}{|c|}{$50.8 \pm 9.39$} & \multicolumn{2}{|c|}{$51.2 \pm 8.99$} & St $\mathrm{t}=0.31$ & 0.76 \\
\hline
\end{tabular}

Table (5) Relation between CHA2DS2-VASc score and LA strain.

\begin{tabular}{ccccccc}
\hline \multirow{2}{*}{ CHADSVASC score } & \multicolumn{2}{c}{$\mathbf{0 - 1}(\mathbf{8 4})$} & \multicolumn{2}{c}{$\geq \mathbf{2}(\mathbf{1 1 6})$} & \multirow{2}{*}{ Statistical test (MW) } & \multirow{2}{*}{ P value } \\
\cline { 2 - 5 } & Median & IQR & Median & IQR & & \\
\hline LA strain (\%) & 30 & $21-34$ & 16 & $14-20$ & 9.34 & $<0.001^{* *}$ \\
\hline
\end{tabular}

Table (6) Validity of LA stain (\%) in prediction of stroke occurrence.

\begin{tabular}{|c|c|c|c|c|c|c|}
\hline \multirow{2}{*}{ LA Strain (\%) } & \multicolumn{2}{|c|}{ Cases with stroke (100) } & \multicolumn{2}{|c|}{ Cases without stroke (100) } & \multirow{2}{*}{ Statistical test $\left(\mathrm{x}^{2}\right)$} & \multirow{2}{*}{$P$ value } \\
\hline & No & $\%$ & No & $\%$ & & \\
\hline$\leq 22.5$ & 82 & 82 & 40 & 40 & & \\
\hline$>22.5$ & 18 & 18 & 60 & 60 & 37.07 & $<0.001^{* * *}$ \\
\hline AUC (95\% CI) & \multicolumn{6}{|c|}{$0.665(0.59-0.74)$} \\
\hline Cut of point & \multicolumn{6}{|c|}{22.5} \\
\hline Sensitivity & \multirow{2}{*}{\multicolumn{6}{|c|}{82}} \\
\hline Specificity & \multirow{2}{*}{\multicolumn{6}{|c|}{$\begin{array}{c}60 \\
67.2\end{array}$}} \\
\hline PPV & & & & & & \\
\hline NPV & \multicolumn{6}{|c|}{76.9} \\
\hline Accuracy & \multicolumn{6}{|c|}{71} \\
\hline
\end{tabular}

Regression analysis showed that the most important parameter to predict stroke risk in atrial fibrillation

Table (7) Regression analysis. patient is LA strain by speckle tracking echocardiography Table (7).

\begin{tabular}{lcccc}
\hline & \multirow{2}{*}{$\boldsymbol{E x p}(\mathbf{B})$} & Sig. & \multicolumn{2}{c}{$\mathbf{9 5 \%} \mathbf{C}$ I for EXP(B) } \\
\cline { 4 - 5 } HTN & .238 & .002 & .096 & .591 \\
DM & 1.244 & .677 & .446 & 3.474 \\
SBP & 1.004 & .777 & .978 & 1.030 \\
Pulse & 1.060 & .003 & 1.021 & 1.101 \\
LA strain $(\%)$ & 51.725 & .000 & 12.217 & 219.005 \\
Transverse diameter & .931 & .075 & .860 & 1.007 \\
MINLA volume & 1.005 & .838 & .957 & .055 \\
LAEF & .869 & .000 & .812 & .931 \\
CHADAVASC Score & .055 & .000 & .012 & .244 \\
\hline
\end{tabular}




\section{Discussion}

The essential relentless atrial fibrillation $(\mathrm{AF})$ is the most widely recognized continued arrhythmia in everyone; its predominance increments with age and is for the most part connected with expanded mortality [10].

Atrial fibrillation brings about basic, hemodynamic and electrical results [1]. Patients with diligent AF have a significant danger of stroke, which is altered by the nearness or the nonappearance of a few hazard factors [2]

As counteraction of stroke/foundational embolism is by a wide margin the most significant part of clinical administration of atrial fibrillation (AF), an approved CHA2DS2-VASc score framework is presently suggested as a Class $1 \mathrm{~A}$ sign for hazard appraisal of stroke [3].

In this investigation, patients were ordered into 2 gatherings, Group I which included 100 patients with steady AF muddled with stroke and Group II that included 100 patients with tenacious $\mathrm{AF}$ not convoluted with stroke (control gathering).

The point of this examination was to evaluate the connection between's left atrial capacity by spot following and stroke hazard in understanding with industrious atrial fibrillation.

Correlation between the 2 gatherings was done as with respect to tolerant socioeconomics, history, clinical introduction, Echocardiography boundaries.

Age is additionally an autonomous hazard factor for stroke. This was meant in the milestone Atrial Fibrillation Investigators (AFI) mapping which recognized age as an indicator of stroke in $\mathrm{AF}$ patients. The age-related increment in the danger of stroke isn't explicit to sex and is watched both in guys and females [11].

Age is certainly not a static however a unique hazard factor for AF-related stroke, and hazard increments from 65 years and upwards. It is a nonstop factor, making it hard to build up a self-assertive cut-off for chance definition purposes [12].

In the current examination, as respect the segment information, there was no factually huge distinction between the 2 gatherings as in regards to age and sexual orientation (58 (50-68) years versus 57.5 (49-65) year, $\mathrm{P}$ esteem > 0.16) Table (1).

This was like Karolina et al. [13] study which uncovered that the two subgroups were comparable with respect to segment qualities. Likewise, Abed Elaziz et al. (14) who examined the relationship between left atrial strain and the CHA2DS2-VASc chance score in patients with atrial fibrillation and found no huge contrast between patients as respect age.

Rather than Saha et al. [15] who considered worldwide left atrial strain and its connection with CHADS2 chance score in patients with atrial fibrillation, discovered huge contrasts between patients as respect age.

This could be clarified by the nearness of other co morbidities as diabetes, hypertension, cardiovascular breakdown and many hazard factors in patients with sinus mood in the current investigation.

In the current examination, no factually critical contrast was found between the 2 gatherings as with respect to the sex (P esteem > 0.05) Table (1) .
This was like Abed Elaziz et al. [14] who found that the sex proportion was comparative between patients of $\mathrm{AF}$.

As opposed to Shaikh et al. [16] who considered spot echocardiographic left atrial strain and solidness file as indicators of atrial fibrillation. They revealed that patients with $\mathrm{AF}$ were bound to be men $(\mathrm{p}<0.05)$.

As indicated by Delia et al. [17] who examined prognostic effect of atrial fibrillation in intense coronary condition, they found that patients with atrial fibrillation were bound to be female ( $\mathrm{p}<0.001)$. This could be clarified by the distinction in clinical attributes between the considered gatherings.

In the current examination, patients with previous history of hypertension and diabetes were progressively pervasive in patients with bunch I contrasted and bunch II patients. (55 versus $25, \mathrm{p}=.001$ and 45 versus $25, \mathrm{p}=.003$, individually).

Olsen et al. [18] in his examination demonstrated that AF was progressively basic in patients with hypertension, diabetes, and more seasoned age, which is steady with our results.Originally, these expectation models were built for anticipating ischemic stroke in patients with $\mathrm{AF}$, yet given the mutual hazard factors for creating stroke and $\mathrm{AF}$, it appears to be similarly equipped for foreseeing $\mathrm{AF}$.

Additionally, Abed Elaziz et al. [14] detailed that DM and HTN were altogether expanded in patients with AF, "36\%" of the patients with $\mathrm{AF}$ were diabetic and "83\% "were hypertensive.

Additionally, Saha et al. [15] revealed that HTN was essentially expanded in patients with $\mathrm{AF}$ as "33\%" patients of $\mathrm{AF}$ were hypertensive and "13\%" patients were diabetic.

In the ebb and flow study, there was no critical measurable contrast between the two gatherings in regards to number of patients with previous history of cardiovascular breakdown, fringe vascular ailment. (50 versus $55, \mathrm{p}=.48$ and 25 versus 30 , $\mathrm{p}=.53$, individually) Table (2).

So also, Abed Elaziz et al. [14] revealed that more patients with $\mathrm{AF}$ had past history of cardiovascular breakdown and fringe vascular malady, 3\%"of the patients with $\mathrm{AF}$ had previous history of stroke, "36\% " had previous history of cardiovascular breakdown and "43\%" had previous history of fringe vascular illness.

This was like Delia et al. [17] who found that previous history of stroke, cardiovascular breakdown and fringe vascular malady were altogether expanded in patients with newbeginning and past $\mathrm{AF}$.

In the current examination pulse and systolic circulatory strain (SBP) were altogether expanded in patients of gathering 1 ( 84 versus 77.5 bpm, $p=.003$ and 125 versus $120 \mathrm{mmHg}, \mathrm{p}$ $=.018$, separately)

There was no huge factual distinction between the two gatherings with respect to diastolic circulatory strain (DBP) (70 versus $70 \mathrm{mmHg}, \mathrm{p}=.194$ ) Table (2).

This was steady with Delia et al. [17] who found that patients with new beginning and past $\mathrm{AF}$ had an altogether more noteworthy pulse. No distinctions were found in the systolic circulatory strain.

In the current investigation, concerning echocardiographic discoveries, Transverse LA distance across and LA insignificant volume were altogether expanded in patients of gathering I contrasted and bunch II patients (4.6cm (4.4-4.88) 
vs.4.5cm (4-4.7), $\mathrm{p}<0.001$ and $41 \mathrm{~cm}(38.25-46)$ versus 39.5 $\mathrm{cm}$ (33-44.75), $\mathrm{p}=.028$, individually).

In the investigation by Sabry et al. [19], AF patients with stroke were bound to have higher LA breadths (anteroposterior, longitudinal, transverse) and LA volumes (maximal and negligible). These finding are likewise in concurrence with Saha et al. (15) who detailed that LA volumes (maximal and negligible) were altogether expanded in AF patients with stroke. Likewise, Habibi et al. [20] found that LA measurements and volumes were fundamentally expanded in AF patients with stroke

So also, Habibi et al. [20] who examined the relationship of left atrial capacity and left atrial improvement in patients with atrial fibrillation likewise found that LA widths and volumes were essentially expanded in AF patients with stroke. In agreement, Deepak et al. [21] who considered the left atrial structure and capacity in atrial fibrillation found that LA breadths and volumes were altogether expanded in AF patients with stroke.

Abdel Rahman et al. [22] who considered expanded left atrial solidness in patients with atrial fibrillation identified by left atrial dot following echocardiography found that LA measurements \&volumes were essentially expanded in $\mathrm{AF}$ patients with stroke.

Our investigation uncovered that LA purging portion was altogether decreased in patients of gathering I contrasted and bunch II patients (29.5 (26-34.75) versus 35.5 (30.25-45), $\mathrm{P}<$ 0.001) Table (3). Online with our investigation, Sabry et al. [19]and Saha et al. [15] found that the LA purging part was fundamentally diminished in AF patients with stroke.

In the current study, Left ventricular end diastolic volume (LVEDV) and left ventricular end systolic volume (LVESV) were of no significant difference in both groups (131.5 (121.25-156.75) vs. $143 \mathrm{ml}$ (99$152.25), \mathrm{p}=0.58$ and 52(38.75-55.75), vs 50 (44-64), $\mathrm{p}$ $=.34$, respectively). Also, Left ventricular ejection fraction (LVEF) was of no significant difference in patients of group I compared with patients of group II (50.8 \pm 9.39 vs. $51.2 \pm 8.99, \mathrm{p}=.76)$ Table( 4$)$.

As to standard echocardiographic boundaries and our outcomes, earlier examinations by Karolina et al. [13] and Kleemann et al. [23] have demonstrated that LVEF $<40 \%$ ensnares the nearness of clots; our outcomes affirm these perceptions.

Ahmed et al. [24], who contemplated the appraisal of left chamber mechanical capacity by misshapening imaging in atrial fibrillation and its connection with CHA2DS2-VASc chance score and found that left ventricular launch division was fundamentally decreased in AF patients with stroke.

This was like Saha et al. [15] who revealed that left ventricular launch portion was altogether diminished in patients with AF.

In the current investigation, Systolic left atrial strain was essentially diminished in patients of gathering I contrasted and patients of gathering II (8.9 (7.78-9.85) versus 14.5 (12-18.5), $\mathrm{p}<0.001)$ Table (2).
This was in concurrence with Sabry et al (19) and Shaikh et al. [16] who found that systolic LA strain was altogether diminished in AF patients with stroke.

Also, Sarvari et al. [25] who contemplated strain echocardiographic evaluation of left atrial capacity predicts repeat of atrial fibrillation. They found that systolic LA strain was fundamentally diminished in AF patients with stroke.

As to top E was of no huge contrast in patients with bunch I contrasted and bunch II patients (75 (65-83.25) vs76.5 (65-83.75), early diastolic mitral annular speed (E") $(7.75$ (6.93-8.48) versus $8(7-8.6), p=0.67)$ Table (2).

This was like Shaikh et al. [16] who detailed that mitral E/e" proportion was essentially expanded in $\mathrm{AF}$ patients with stroke. Additionally, Acar et al. [10] who examined the commonness and indicators of atrial fibrillation in hemodialysis patients and found that mitral E/e" proportion was altogether expanded in AF patients with stroke.

In the current investigation, there was noteworthy diminishing in LA strain with in patients with CHA2DS2-VASc score $>2$ contrasted with patients with CHA2DS2-VASc score < 2 (16 (14-20) versus 30 (2134), p <.001) Table (3).

Islas et al. [26] and Kurosawa et al. [27] expressed that LA strain was connected fundamentally and autonomously with CHA2DS2-VASc score and these discoveries are with our outcomes.

On the opposite side, in the examination by Ahmed et al. [24] , there was a non-huge relationship between's CHA2DS2 VASc score and pinnacle LA strain. Likewise, there was a non-noteworthy distinction and furthermore there was a non-huge relationship between's left atrial strain and CHA2DS2 VASc score in persistent with score not exactly or equivalent to 2 or those with score in excess of 2 which check our investigation results.

In the current investigation, When estimations of the systolic LA strain, tissue Doppler imaging(systolic (S") and early diastolic (E") mitral annular speeds), systolic LA myocardial velocity(s) and left ventricular launch portion (LVEF) corresponded with the CHA2DS2-VASc score, there was a negative connection, however it didn't arrive at factual criticalness $(\mathrm{P}>0.05)$. The relationship between's Left atrial purging division (LAEF) and CHA2DS2-VASc score was noteworthy and negative ( $\mathrm{p}$ $<0.05)$ Tables (6).

Sabry et al. [19] study uncovered that systolic LA strain, systolic (S) and early diastolic (E") mitral annular speeds, systolic LA myocardial velocity(s) and left ventricular EF were adversely connected with theCHA2DS2-VASc score however without factual centrality and the relationship between's LA exhausting portion and CHA2DS2-VASc score was huge and negative.

The connection between's the CHA2DS2-VASc score and LA distances across, LA volumes, Peak E, mitral E/e" proportion and early diastolic myocardial 
speed (e) in patients with AF was sure, yet it didn't arrive at measurable centrality which is like our outcomes.

Likewise these outcomes was like Abed Elaziz et al. [14] who found that the connection between's the CHA2DS2-VASc score and the pinnacle LA strain was negative relationship, yet it didn't arrive at measurable criticalness.

Likewise, Islas et al. [26] who examined the thromboembolic hazard in atrial fibrillation and found that LA strain was corresponded fundamentally and autonomously with CHA2DS2-VASc score. Nonetheless, Kurosawa et al. [27] who examined the connection between left atrial strain and CHA2DS2VASc score contrasted with left atrial member discharging stream speed found that the segments of the score were not altogether corresponded with LA strain aside from age and CHF which were freely related with LA strain.

The CHA2DS2VASc score is an alteration of the CHADS2 score that expects to improve the forecast of stroke chance in patients with atrial fibrillation. Patients with a high CHA2DS2VASc score have a high rate of ischemic stroke in spite of getting anticoagulation treatment [28].

In our investigation, there was huge lessening in LA strain in patients with CHA2DS2-VASc score > 2 contrasted with patients with CHA2DS2-VASc score $<2$ (16 (14-20) versus 30 (21-34), p <.001) Table (5).

Not at all like the examination by Ahmed et al. (24) who found no huge connection between's left atrial strain and CHA2DS2 VAS c score in persistent with score not exactly or equivalent to 2 or those with score more than 2.

In the examination by Sasaki et al. [28] they found that, LAA brokenness was related with expanding CHA2DS2VASc score. The finding that there is a connection between CHA2DS2VASc score and LA strain is steady with past reports, which proposed that LA strain is associated with the danger of thromboembolism as characterized by CHADS2 score $[15,29,30]$.

Our investigation uncovered that, the collector administrator trademark (ROC) bend was utilized to test symptomatic estimation of different echocardiographic boundaries models in the connection between's left atrial capacity by dot following and stroke hazard in patients with constant atrial fibrillation and demonstrated that the estimation of LA strain $<22.5$ was appeared to have the best analytic precision with (affectability $=82 \%$; particularity $=60 \%$ ) in foreseeing the stroke chance in atrial fibrillation understanding with AUC.871 Table (6).

Upon ROC bend investigation in the examination by van sanctum Berg et al. [31], they discovered great symptomatic execution for LA positive longitudinal strain in the four-chamber see (ideal cutoff, $\leq 6.7 \%$; $\mathrm{AUC}=0.84 ; \quad$ sensitivity $=77.1 \% ; \quad$ specificity $=77.1 \%)$, which recommended that the indicative execution for LAAT recognition in patients with ceaseless non valvular AF might be higher when utilizing the mix of such LA-based boundaries than when utilizing the CHA2DS2-VASc score alone. Additionally, they found that the CHA2DS2-VASc score was not related with LAAT, which is in concurrence with the discoveries of Sugiura et al. [32] and Tang et al. [33] however all are can't help contradicting our outcomes.

These outcomes can be clarified by the way that, in incessant nonvalvular AF, LA dilatation and brokenness is the essential supporter of cluster arrangement [31]. Different creators likewise proposed models joining clinical and echocardiographic boundaries. For instance, Obokata et al. [1] proposed models that joined CHA2DS2-VASc score, oral anticoagulant use, left ventricular discharge division, and LA all out longitudinal strain to increment prescient worth.

The upside of the models proposed in our current investigation is that they accomplish great prescient capacity utilizing just echocardiographic boundaries, in this way being simpler to apply in clinical practice.

In the examination by Sabry et al. [19], the estimation of systolic LA strain $\leq 17.44$ was appeared to have the best demonstrative exactness (affectability = $42.11 \%$; specificity $=62.07 \%$ ) in foreseeing the nearness of CHA2DS2 - VASc $\geq 2$.

In another examination by $\mathrm{Li}$ et al. [34], the beneficiary administrator trademark (ROC) bend was utilized to test indicative estimation of different echocardiographic boundaries models in anticipating AF of CHA2DS2-VASc score however shockingly, the distinction didn't show up at the factual centrality.

As indicated by the relapse examination, the most significant boundary to anticipate stroke hazard in atrial fibrillation persistent is LA strain by spot following echocardiography Table (7).

Not at all like the ongoing investigation by Wang et al. [35], they didn't see the CHA2DS2-VASc score as altogether connected with LAA brokenness, and this score was not a perfect stage to foresee dangers related with stroke utilizing the LAT esteem as a comparator. This irregularity was additionally seen in past investigations [36, 37].It is clear that the scoring system includes an assessment of the risks of early stroke, including those that cause vascular wall injury and hypercoagulable status [38]. However, the scoring system did not analyze the effect of AF itself on cardiac remodeling which is a continuous present risk factor for stroke. Other possible variations include AF duration, structural cardiac abnormalities, race, anticoagulation adequacy, and other cardiovascular risk factors that might be associated with the observed effects.

\section{This study has some limitations}

1- Despite the fact that we did our best to obtain only good quality echocardiographic images, still some images are suboptimal affecting.

2- Strain images measured by speckle tracking echocardiography.

3- The duration of follow up was limited. 


\section{Conclusion}

It is inferred that the LA strain, which is a marker of dynamic LA work, is fundamentally diminished in AF patients.

Additionally, Speckle following examinations of the left chamber may offer significant data on which patients would profit the most from delayed heart checking for distinguishing proof of PAF.

\section{Recommendation}

We recommended to do this study on large number of patients to confirm our study results. Also, we recommended to use the speckle tracking analyses of the left atrium to get its benefits for predicting of stroke in AF patients.

\section{References}

[1] M. Obokata, K. Negishi, K. Kurosawa, Left atrial strain provides incremental value for embolism risk stratification over CHA2DS2-VASc score and indicates prognostic impact in patients with atrial fibrillation. J., of the American Society of Echocardiography, Vol. 27(7), PP. 710-716,2014.

[2] V. Fuster, L. Rydén, D. Cannom, ACC/AHA/ESC 2006 Guidelines for the Management of Patients with Atrial Fibrillation: a report of the American College of Cardiology/American Heart Association Task Force on Practice Guidelines and the European Society of Cardiology Committee for Practice Guidelines (Writing Committee to Revise the 2001 Guidelines for the Management of Patients With Atrial Fibrillation): developed in collaboration with the European Heart Rhythm Association and the Heart Rhythm Society. Circulation ,Vol. 7(114), PP. e257354,2006.

[3] P. Kirchhof , A. Auricchio, J. Bax, Outcome parameters for trials in atrial fibrillation: executive summary. Recommendations from a consensus conference organized by the German Atrial Fibrillation Competence NETwork (AFNET) and the European Heart Rhythm Association (EHRA). Eur. Heart J , vol, 28, PP. 28032817,2007.

[4] G.Y. Lip, R. Nieuwlaat, Refining clinical risk stratification for predicting stroke and thromboembolism in atrial fibrillation using a novel risk factor-based approach: the Euro Heart Survey on Atrial Fibrillation. Chest, Vol. 137, PP. 263-272,2010.

[5] G. Perk, P.A. Tunick , I. Kronzon , Non-Doppler two-dimensional strain imaging by echocardiography from technical considerations to clinical applications. J Am Soc. Echocardiography, Vol. 20, PP.234-243, 2007.

[6] R.M. Lang, M. Bierig , R.B. Devereux , Recommendations for chamber quantification: a report from the American society of echocardiography's guidelines and standards committee and the chamber quantification writing group, developed in con-junction with the European association of echocardiography. A branch of the European society of cardiology. J., of the American Society of Echocardiography, vol 12(18), PP. 1440-1463,2005.

[7] N.B. Schiller, J.S. Simonson, Descent of the base of the left ventricle. An echocardiographic index of left ventricular function.J Am SocEchocardiogr, Vol.2(1), PP. 25-35, 1989.

[8] L. Thomas, K. Levett, A. Boyd, Changes in regional left atrial function withaging: evaluation by doppler tissue imaging.European J.,of Echocardiography, Vol.2(4), PP.92-100, 2003.

[9] G. Salvo, P. Caso , R. Piccolo, Atrial myocardial deformation properties predict maintenance of sinus rhythmafter external cardio version of recent-onset lone atrial fibrillation: a color Doppler myocardial imaging and transthoracic andtransesophageal echocardiographic study. Circulation, Vol.3(112), PP. 387-395, 2005.

[10] G. Acar, A . Akçay, E. Dogan, The prevalence and predictors of atrial fibrillation in hemodialysis patients. Turk Kardiyol Dern Ars, Vol. 38, PP. 813, 2010.

[11] Z. Zafraan, K. Anne, M.F. Ameenathul, Atrial Fibrillation in Older People: Concepts and Controversies. Front Med (Lausanne), Vol. 6, PP. $175,2019$.

[12] O. Oreoluwa , N. Ifeanyi , Stroke risk stratification in atrial fibrillation: a review of common risk factors. J Community Hosp Intern Med Perspect, Vol.9(2), PP. 113-120,2019.

[13] K. Karolina, W.M. Blazej , M. Dawid, Association between left atrial function assessed byspeckle-tracking echocardiography and the presence of left atrial appendage thrombus in patients with atrial fibrillation. Anatol J Cardiol , Vol. 18, PP. 15-22, 2017

[14] W.F. Abed Elaziz, M.K. Ahmed, M.A. Nasif , The association between left atrial strain and the CHA2DS2-VASc risk score in patients with atrial fibrillation. Menoufia Medical J.,, Vol. 27, PP. 699-704, 2014.

[15] S.K. Saha, P.L. Anderson, G. Caracciolo, Global left atrial strain correlates with CHADS2 risk score in patients with atrial fibrillationJ. Am. Soc. Echocardiogrvol ,Vol. 24, PP. 506-512,2011.

[16] A.Y. Shaikh, A. Maan, U.A. Khan, Speckle echocardiographic left atrial strain and stiffness index aspredictors of maintenance of sinus rhythm after cardioversion for atrial fibrillation: a prospective study. Cardiovascular Ultrasound, Vol. 10, PP.48,2012.

[17] M.A. Delia, M.J.V. Caballero, J.C.G. Rubira, Prognostic impact of atrial fibrillation in acute coronary syndromes results from theARIAM registry. European Heart J.,: Acute Cardiovascular Care, Vol. 3(2) , PP.141-148, 2014. 
[18] F.J. Olsen, J. Sune, S. Jan, Global longitudinal strain predicts incident atrial fibrillation and stroke occurrence after acute myocardial infarction, Medicine: November, Vol. 95, PP.44 p e5338, 2016.

[19] A.M. Sabry, H.M. Abo El Enin, M.A. Hamoda, et al: The Correlation between Left Atrial Deformation Indices andthe CHA2DS2 - VASc Risk Score in Patients with Atrial Fibrillation. J Clin Exp Cardiolog, Vol.35,PP.7- 425,2016.

[20] M. Habibi, A.C. Joao, L.Z. Stefan, Association of Left Atrial Function and Left Atrial Enhancement in Patients with Atrial Fibrillation by Cardiac Magnetic Resonance Study. JACC, Vol.6(63),PP.493-505, 2014.

[21] K.G. Deepak, M.S. Amil, P.G. Robert, et al: Left atrial structure and function in atrial fibrillation: ENGAGE AF-TIMI 48and for the Effective aNticoaGulation with factor $x A$ next GE neration in AF-Thrombolysis In Myocardial Infarction 48 (ENGAGE AF-TIMI 48) Echocardiographic Study Investigators. Eur Heart J,Vol.35(22),PP.1457-65,2014.

[22] H. Abdel Rahman, A.K.M. Hassan, A.H.S. Abosetta, et al: Increased left atrial stiffness in patients with atrial fibrillation detected by left atrial speckle tracking echocardiography. The Egyptian Heart J.,, Vol.3(67),PP.217-223,2015.

[23] T. Kleemann, T. Becker, M. Strauss, Prevalence and clinical impact of left atrial thrombus and dense spontaneous echo contrast in patients with atrial fibrillation and low CHADS2 score. Eur $\mathbf{J}$ Echocardiogr, Vol.10, PP.383-8,2009.

[24] M.K. Ahmed, W.F. Abed Elaziz and M.A. Nasif (): Assessment of left atrium mechanical function by deformation imaging in atrial fibrillation and its correlation with CHA2DS2-VASc risk score. The Egyptian Heart J.,, Vol.3(67),PP.209$215,2015$.

[25] S.I. Sarvari, K.H. Haugaa, T.M. Stokke, et al. (): Strain echocardiographic assessment of left atrial function predicts recurrence of atrial fibrillation. European Heart J., Cardiovascular Imaging, Vol.10(3),PP.109-117,2015.

[26] F. Islas, C. Olmos, M. Paiva, Thromboembolic risk in atrial fibrillation: association between left atrium mechanics and risk scales. A study based on 3DWMT. Eur. Heart J, Vol. 34,PP.20-26,2013.

[27] K. Kurosawa, K. Negishi, R. Tateno, et al: Relationship left atrial strain and CHA2DS2VASc score compared to left atrial appendage emptying flow velocity, Eur Heart J,Vol.34(1),PP.20-24,2013

[28] S. Sasaki, T. Watanabe, H. Tamura, Left atrial strain as evaluated by two-dimensional speckle tracking predicts left atrial appendage dysfunction in patients with acute ischemic stroke. BBA Clinical, Vol.2,PP. 40-47,2014.

[29] G.V. Naccarelli, M.P. Panaccio, G. Cummins, et al: TuCHADS2 and CHA2DS2-VASc risk factors to predict first cardiovascular hospitalization among atrial fibrillation/atrial flutter patients. Am. J. Cardiol, Vol.109,PP.15261533,2012.

[30] J.Y. Shih, W.C. Tsai, Y.Y. Huang, Association of decreased left atrial strain and strain rate with stroke in chronic atrial fibrillation. J. Am. Soc. Echocardiogr,Vol.24, PP. 513-519,2011

[31] N.W. van den Berg, J. Neefs, W.R. Berger PREventive left atrial appenDage resection for the predICtion of fuTure atrial fibrillation: design of the PREDICT AF study. J., of Cardiovascular Medicine, Vol.20(11),PP.752-61,2019.

[32] S. Sugiura, E. Fujii, M. Senga, Clinical features of patients with left atrial thrombus undergoing anticoagulant therapy. J., of Interventional Cardiac Electrophysiology;Vol.34(1),PP.59-63,2012.

[33] R.B. Tang, J.Z. Dong, X.L. Yan, Serum uric acid and risk of left atrial thrombus in patients with nonvalvular atrial fibrillation. Canadian J., of Cardiology,Vol.30(11),PP.1415-1421,2014.

[34] Y. Li, W. Ding and H. Wang, Relationship of CHA2DS2-VASc and CHADS2 Score to Left Atrial Remodeling Detected by Velocity Vector Imaging in Patients with Atrial Fibrillation. J., pone,Vol.10(8),PP.76-53,2013..

[35] Y. Wang, M. Li, L. Zhong, et al. Left Atrial Strain as Evaluated by Two-Dimensional Speckle Tracking Predicts Left Atrial Appendage Dysfunction in Chinese Patients with Atrial Fibrillation. Cardiology Research and Practice,Vol.33,PP.1-8,2020.

[36] R. Beigel, N.C. Wunderlich, S.Y. Ho, et al: The left atrial appendage: anatomy, function, and noninvasive evaluation. JACC: Cardiovascular Imaging,Vol.7(12),PP.1251-1265,2014.

[37] H. Yarmohammadi, B.C. Varr, S. Puwanant, et al. (): Role of CHADS2 score in evaluation of thromboembolic risk and mortality in patients with atrial fibrillation undergoing direct current cardioversion (from the ACUTE Trial Sub study). The American J., of Cardiology,Vol.110(2),PP.222-226,2012.

[38] J. Huang, S.L. Wu, Y.M. Xue, et al: Association of CHADS2 and CHA2DS2-VASc scores with left atrial thrombus with nonvalvular atrial fibrillation: a single center based retrospective study in a cohort of 2695 Chinese subjects. Bio Med Research International, Article ID ,Vol.6,PP.839-589,2017. 